\title{
UNA REFLEXIÓN CRÍTICA DESDE LA EDUCACIÓN INTERCULTURAL HACIA LA PEDAGOGÍA DE LA RECREACIÓN
}

\author{
A CRITICAL REFLECTION FROM INTERCULTURAL EDUCATION \\ TO THE PEDAGOGY OF RECREATION
}

\author{
Miguel Ángel Alomía Riascos ${ }^{1}$ \\ En educación intercultural es posible argumentar que al relacionar las estructuras, \\ con las interacciones que generan, o pueden generar tales estructuras y se establecen \\ procesos de retroacción consecuencia de tales interacciones, todo se despliega en torno a la holocomplejidad.
}

\section{Resumen}

El presente escrito tiene como propósito considerar con un enfoque de orientación, dinamización y puesta en acción el paradigma de la interculturalidad, a partir de algunos roles (tareas) asumidos, o por asumirse, por parte de determinadas prácticas educativas recreativas que debieran ser incluyentes mediante procesos de formación, aprendizaje e implementación. Estos se dan en marcos de realidad sociocultural - la diversalidad-y debieran estar presentes tanto en el ámbito escolar, como el extraescolar (actividades recreo-deportivas) y sin excepción en contextos de tiempo libre (prácticas de ocio). De esta manera se constituyen en ejercicios de prácticas emancipadoras, respetuosas, justas, solidarias y humanas provocando fructíferas relaciones, correlaciones e interacciones siempre presentes en los ambientes culturales, educativos, escolares y comunitarios, en consonancia con una perspectiva intercultural.

Palabras claves: interculturalismo, educación, recreación, transformación social, organización, pedagogía de la recreación.

\section{Abstract}

The purpose of this article is to consider an approach to guidance, promotion and put into action the paradigm of interculturalism from some roles (tasks) incurred or assumed by certain recreational educational practices that should be inclusive through training processes, learning and implementation, given in frames socio-cultural - diversity - that must be present, both, in schools, as extra school (recreo-deportivas activities) without exception in the context of free time (leisure practices) becoming in exercises of emancipation, respectful, fair, solidarity and human causing successful relations, correlations and interactions that are always present in the cultural, educational, school and community settings in agreement with intercultural practices.

Keywords: Interculturalism, Education, Recreation, Social transformation, Organization, Pedagogy of recreation.

Fecha de recepción: 15 de mayo de 2015

Fecha de aprobación: 1 de octubre de 2015

Para citar este artículo:

Alomía, M.A. (2015). Una reflexión crítica desde la educación intercultural hacia la

Pedagogía de la Recreación. Lúdica Pedagógica, (22), 45-53.

1 Profesor de planta, Facultad de Educación Física, Universidad Pedagógica Nacional. Correo electrónico: malomia@pedagogica.edu.co 


\section{INTRODUCCIÓN}

En el presente artículo se pretende desencadenar múltiples emociones que conlleven al abordaje de elaboraciones conceptuales y prácticas de transformación sustentable, esto es, respeto a la vida; que es múltiple y diversa implicando para ello la educación, la recreación y la educación recreativa, es decir, pretende formular un marco de fundamentación epistemológica conducente a una pedagogía de la recreación a partir de su incidencia relacional formativa, socioeducativa y cultural en lo individual, grupal y comunitario, lo cual provoca a tener una consideración otra, emergente de la interculturalidad con perspectiva holocompleja.

Su enunciación se inscribe en la naturaleza y políticas de la Universidad Pedagógica Nacional, la Facultad de Educación Física, entidad fundadora en relación con la docencia en el campo, gracias a sus programas de pregrado y posgrado, los desarrollos y alcances de los grupos y trabajos de investigación, los aportes efectuados por el gremio de profesionales y la comunidad académica, y además, por el entorno relacional resultante de nuevos retos, necesidades y nuevas realidades sociales ciudadanas y del saber pedagógico específico.

\section{LA NECESIDAD DE UNA POSTURA EMERGENTE}

En procura de alterar determinados arraigos de la cultura hegemónica, excluyente y colonialista — con respecto a legados establecidos por herencias generacionales, que se presentan en sociedades eminentemente patriarcales y deterministas, donde la mayor parte de sus estructuras dan cuenta de pensamientos de empoderamiento y sometimiento desde los cuales las sociedades se constituyeron (Maturana y Varela, 2003) — se busca reconocer, relacionar y convocar dinámicas de reflexión y abordaje conjuntas, con aquellas capas poblacionales que tradicionalmente han estado excluidas de procesos de participación, construcción y disfrute de derechos.

Es necesario tener en cuenta los siguientes aspectos: múltiples realidades sociales, políticas y culturales están configuradas y fijadas por las instituciones, máximo invento del pensamiento patriarcal, en donde se genera una amplia variedad de organizaciones que, de una u otra manera, excluyen y someten en un determinado orden o lineamiento a personas, grupos y comunidades mediante la aculturación, o endoculturación, impuesta por la comunidad hegemónica, un ejemplo de ello lo constituye el problema de género.

Así, un abordaje crítico reflexivo desde el hecho educativo, recreativo dialógico e incluyente debe generar unas dinámicas relacionales con la problematización de posibles vínculos y componentes de realidad (análisis crítico del discurso) ${ }^{2}$, de los que emerge, de acuerdo con teorías de las Ciencias Sociales, para los procesos de cambio sociocultural en una concepción contemporánea de pedagogía crítica. Esta aparece como tal, para superar las carencias del concepto amplio del campo educativo, pero que fue necesario aplicar en la especificidad de la educación en general, haciéndose más incluyente. Lo anterior permite - de diversas maneras - apropiarse y tomar con responsabilidad una serie de aproximaciones por la formulación de un agregado de preguntas.

Es pertinente, desde la perspectiva emergente y compleja de la interculturalidad, considerar que esta relaciona, en una multiplicidad de aspectos, las diversidades, lo dialógico, la equidad y la colaboración, entre otros, para adelantar procesos de reflexión en torno a la pedagogía de la recreación mediante el abordaje de cuestionamientos como: ¿De qué modos ocurre hoy la aceptación y toma de conciencia del valor educativo de la recreación? ¿Educativamente, qué es la recreación, por qué se gestionan eventos, presupuestos, recursos específicos y campañas publicitarias acerca de ellas a nivel formativo, cultural, institucional y comunitario; como sucede en ferias, carnavales, festivales, promoción (prensa) o espectáculo, a diferencia de otros ámbitos de desempeño laboral y profesional docente? ¿Quiénes se interesan porque lo anterior suceda y para qué? ¿Cuáles vínculos traza la recreación entre la educación, la empresa, las organizaciones y la política? ¿Qué hacer con la recreación en la escuela en particular y en el ámbito educativo en general y para qué? ¿Qué debe hacer el profesional de la docencia, la familia, la escuela, la comunidad y las organizaciones sociales en procura de la ampliación y desarrollo del campo?

2 Es un enfoque interdisciplinar articulado al estudio del discurso, la argumentación que relaciona el "lenguaje como una forma de práctica social" y analiza cómo los discursos se reproducen y se resisten mediante los procesos de dominación. 
Así, con Sandra Harding ${ }^{3}$ (1996) desde/para/con los estudios tanto culturales, interculturales, sociales (masculinos, femeninos) como humanísticos, vale la pena hacer la siguiente afirmación: "Hemos organizado nuestros mundos social y natural en términos de significados de género, en cuyo contexto se han construido instituciones y significados raciales, de clase y culturales históricamente específicos" (p. 119), los cuales movilizan y dinamizan propósitos. La recreación con perspectiva intercultural se constituye en mediador importante en los procesos de interrelación gilánica ${ }^{4}$.

Lo anterior resulta válido, máxime si a lo largo de la historia de la humanidad, la mayor exclusión y discriminación ha estado dada hacia lo femenino, entonces, a partir de la organización de la sociedad desde la perspectiva de sistema andrógino, en la que se concentra una estructura jerárquica asignándose el hombre la función de proveedor, protector y sobre todo definidor del destino de sus otras integrantes - sus pares femeninos-, de igual manera debe acontecer con las comunidades y grupos marginados más aún, tal empoderamiento se soporta con un referente contextual especifico desde la antigüedad como lo ha sido la religión (sucede así con las más conocidas, populares y numerosas), donde unos dioses todopoderosos establecen desde sus lineamientos que le corresponde al hombre responder por el mantenimiento del orden establecido (Eisler, 1990).

La conformación de una sociedad desde la perspectiva andrógina, patriarcal, falocéntrica se ha desarrollado a través de la creación de instancias que poco a poco dan la estructura de pensamiento lineal y jerárquico, de sometimiento y subyugación, manifiesto en la organización social - hegemónica- Tal organización social se evidencia en la institucionalización de los comportamientos del ser humano - por ejemplo, procreación, supervivencia, convivencia, comunicación,

3 Exdirectora del Centro de Estudio de la Mujer de la Universidad de California, en Los Ángeles (1996-2000), quien ha contribuido al desarrollo de la teoría del punto de vista, siendo una de las fundadoras del campo de la epistemología feminista, así como a los estudios de ciencia, tecnología y género.

4 En relación con Riane Eisler (1990) quien construye el término gilania. Gi se toma de la palabra griega gyne, "mujer"; an de andros, "hombre", conectados con la letra ele, que aporta un doble significado. Desde el tao representa vínculo entre ambas mitades de la humanidad presentes en las leyes de generación y de polaridad, sistémicamente es una connotación más amplia y totalizante que se pueda asumir desde la androcracia o la ginecocracia. afectividad-, los cuales son recogidos y reglamentados a través de las instituciones encargadas de regular, controlar, castigar a los miembros de la comunidad según lo establecido.

Surgen, entonces, instituciones como la familia, el gobierno, la escuela, el ejército, la religión; todos símbolos hegemónicos del poder patriarcal que ha determinado el destino de la humanidad desde hace muchos siglos, como se logra argumentar con Kosko (2000). Estas entidades, por supuesto, tienen como eje central para su funcionamiento principios que tienen que ver con el individualismo, la negación de otras opciones que no se acomoden a sus intereses, etnocentrismo ideológico por ejemplo, el sometimiento de los más débiles entre ellos la mujer considerada como inferior, con menores capacidades y posibilidades y que necesita ser controlada y cuidada, de igual condición sucede con la niñez, la población adolescente y adulta mayor.

Entre sus características se cuenta la de asumir una manera distinta de vivir su cuerpo, un cuerpo sublimado (Boffy Muraro, 2004), con un pensamiento que desde su conformación a través de ser flexible a la percepción, se enriquece y se recrea de manera multifacética ante las distintas situaciones de la cotidianidad, que se despliega y repliega (Wilber, 1987) su opción para la convivencia desde la diversidad, centrada en la búsqueda de la armonía, la equidad, la restitución de su papel en el universo de la humanidad son condiciones que amenazan el eje desde el cual se constituyó por siglos la sociedad y así mismo la cultura dominante y para la cual un enfoque intercultural establece una mejor opción.

\section{REFLEXIONES CRÍTICAS SOBRE LA RECREACIÓN}

En la actualidad se evidencia una marcada infinidad, complejidad y cruzamiento cultural de las prácticas tanto corporales y motrices, como recreativas; tal fenómeno social de importancia, así en sus experiencias lo mismo que en sus campos de desarrollo (conocimiento) amerita un abordaje, estudio e investigación serio y diferente al que se ha dado hasta ahora. Su campo de acción, al igual que ocurrió con las prácticas deportivas en el ámbito escolar, estuvieron pensadas inicialmente para disciplinar e instruir a la juventud, y se extendió luego hacia nuevos grupos generacionales, culturales 
y poblacionales, comprometiendo variados aspectos del ambiente social: económico, productivo, laboral, administrativo, político y cultural.

La importancia social y política de la recreación, en la primera mitad del siglo $\mathrm{xx}$, ha configurado una estructura organizacional y del conocimiento que deriva en la conformación de las llamadas ciencias de la actividad física, como también, aunque de manera mínima, en las ciencias aplicadas del deporte, o para otros, ciencias del deporte. No obstante, el carácter y desarrollo de tales áreas del conocimiento, para el ámbito latinoamericano transcurrió en un ejercicio reproductor de técnicas y procedimientos, muchas veces carentes de fundamento y alejado de los debates, necesidades con sus satisfactores (Max-Neef, 1993) así como, de beneficios teóricos (Sonnenschein, 1980).

La recreación, en cuanto práctica y manifestación cultural (moderna, lineal, positivista, cartesiano-newtoniana), de igual manera, como hecho o fenómeno social, se relaciona en un entramado de realidades complejas - aspecto que no ha sido tratado con rigurosidad de manera sistémica y holística-, caracterizadas por una cosmodimensionalidad múltiple (epistémica, antropológica, filosófica, científica, económica, social, comunicativa, estética, ética, espiritual, educativa y tecnológica), entre muchas por mencionar. Como práctica organizacional (institucional), demanda una estructuración funcional local, regional, nacional e internacional (global) que comprende varios ámbitos en los que involucra un conjunto de sistemas como: el estatal (político-gubernamental), ambiental, económico-financiero, bienestar y desarrollo, cultural, laboral, legal o normativo, determinado a su vez por un conjunto de principios que le confieren valor social y necesidad comunitaria.

Como fenómeno sociocultural, la recreación tiene una especial importancia relacional ${ }^{5}$, de interacción(es) ${ }^{6}$ (Maturana, 2005), individual(es) y colectiva(s); de igual

5 Establecer relaciones, es pasar de la fragmentación a la visión integral, holista y sistémica, lo cual constituye uno de los principios epistémicos fundamentales del paradigma emergente.

6 Probablemente, uno de los principales cambios que se evidencia en los contextos de relación y, por tanto, de aprendizaje se da en las formas como las personas se comunican y establecen relaciones, conllevando a nuevas formas de interacción matizada por una convergencia de medios y tecnologías, y procesos de interacción continua, articulándose en la cotidianidad de cada persona en sus contextos. manera, de retroacción(es) ${ }^{7}$, tanto por las adjudicaciones que los sistemas anteriormente mencionados le otorgan, las peticiones que le hace el Estado, los gustos e intereses poblacionales de utilización y práctica, los beneficios y bondades adquiridas, y las transformaciones estructurales que posibilita.

A pesar de lo anterior (importancia sociocultural), la sistematicidad académica e investigativa existente sobre la recreación resulta insuficiente, por lo menos en los contextos latinoamericano o colombiano, consideración que se formula desde oportunidades, necesidades y fortalezas con las cuales se pueden establecer unos mínimos, unas condiciones y posibilidades de desarrollo; a través de sus implicaciones políticas y sociales para la niñez, la juventud y las personas adultas por las configuraciones sociovalorativas constituidas tanto en grupos, como en comunidades.

Ocuparse de este fenómeno sociocultural implica reconocer desarrollos culturales necesarios de implementación, siendo ineludible la constitución de organismos (organizaciones) que se ocupen de lo teórico-práctico, inclusive que emerjan como estructuras consistentes para potencializar prácticas ocupacionales, reflexivas y profesionales (saberes, conocimientos y producción de conocimiento científico-técnico) de la recreación mediante su robustecimiento; de esta manera se determinan problemáticas que resultan impropias del campo específico en su quehacer, responsable por favorecerla y brindarle un estatus de validación, formalización y desarrollo.

7 Es un proceso relacionado con cambios - o lo que puede ocasionar cambios en los ecosistemas-generando una reacción ante una perturbación. Por tanto, ocurre que una transformación conlleve a variaciones del estado inicial, esta puede ser positiva o negativa. La retroacción positiva es aquella que tiene el efecto específico de amplificar la perturbación. La retroacción negativa tiene el efecto neto de atenuar la perturbación. Ésta última es empleada en varias ciencias y en cada una de ellas su acepción es puntual, por ejemplo: perturbación, en astronomía, se entiende como el conjunto de alteraciones que la órbita de un objeto causa por interacciones gravitatorias con otros cuerpos. En medicina y ciencias de la salud, se llama noxa a todo lo que ingresa al cuerpo humano y genera una perturbación o perjuicio biológico, social o psicológico. Perturbación, en ecología, es un suceso puntual que altera el equilibrio en un ecosistema. Perturbación, para la geología, son cambios que ocurren en la naturaleza de los depósitos aluviales en el tiempo. 


\section{REFLEXIÓN CRÍTICA SOBRE LA PEDAGOGÍA}

La pedagogía y el discurso pedagógico como fenómeno o sistema de complejidad ${ }^{8}$ creciente (Maldonado, 2012), demanda para la comprensión de la educación con perspectiva sistémica ${ }^{9}$ y holística ${ }^{10}$ una transformación en las lógicas de abordaje. Un planteamiento contemporáneo de esta naturaleza (postura paradigmática ${ }^{11}$ de la interculturalidad) genera e invita al establecimiento de un tipo de relación ética en la transición de las relaciones sociales que se producen en/desde/con y para la educación. A la vez que se problematizan y desarrollan los aspectos, elementos y propiedades de la educación, esta y su multiplicidad de prácticas quedan estructuradas organizacionalmente por una postura paradigmática. De acuerdo con Rozengardt (2006), "esto es un poder del que hay que tener conciencia. [Así], los discursos pedagógicos están en disputa. Hay variados y confrontan por ser reconocidos y validados" (s.p.).

Tanto las realidades, como los pensamientos y los conocimientos son complejos; por lo cual se requiere de la complejidad para entender el mundo, puesto que hoy la estructura interna del conocimiento, como tantas otras dimensiones humanas, está sujeta a cambios. En la actualidad se reconoce en diversos escenarios, que vivimos, convivimos y habitamos realidades matizadas por la era de las posibilidades o probabilidades, donde se invalida dar por definitivo e irreversible cualquier tipo

8 Como teoría epistémica se relaciona con el sistema de la complejidad (conjunto de ciencias), conviene mencionar la dificultad para definir con mediana precisión este concepto. En el ámbito investigativo, es indefinible de manera sencilla.

9 La aportada a partir de la teoría general de sistemas (Ludwig von Bertalanffy), y basada en que las propiedades de los sistemas no pueden describirse significativamente en términos de sus elementos separados, su compresión solo se presenta al estudiarse y considerarse globalmente, involucrando la totalidad de sus partes en interdependencia.

10 Entendida como totalidad armónica e integrada, por ejemplo, desde tales posturas resulta válido plantear para la educación física: ¿Cómo se trata, desarrolla y se aplica desde sus ámbitos las continuidades "entre lo social, lo humano y sus raíces biológicas"? (Maturana, 1994).

11 En la búsqueda de decolonizar la interculturalidad, especialmente la postura suramericana bilingüe o la misma europea de matiz español, recurriendo para ello al abordaje del paradigma de la complejidad. El término paradigma puede comprenderse de dos maneras. La primera, desde una dimensión global, es decir, como enfoque, conjunto o sistema cognoscitivo desde el cual se comprende o interpreta la realidad o una parte de ella. En segundo término, desde una concepción particular, que se refiere caso específico en el que se ilustra de forma contundente, a la riqueza comprensiva y explicativa que soporta dicho enfoque. de conocimiento. Si el pasado siglo xx se caracterizó como la época de la búsqueda de certezas científicas y del desarrollo acelerado de las diferentes disciplinas y subdisciplinas, el periodo actual, está llamado a ser el siglo de las incertidumbres, las diversidades donde el multiculturalismo es apenas una de sus manifestaciones, la interdisciplinariedad y transdisciplinariedad, así como la recuperación de la comprensión del conocimiento como totalidad (holística) intercultural.

Al mismo tiempo, el conocimiento se produce en diversos ámbitos, cada vez más próximos a sus escenarios de práctica e implementación, por sus aplicaciones tecnológicas tiende a movilizarse y transformarse con prontitud, en países con altos desarrollos económicos, financieros y tecnológicos, de los recintos acadé-micos; se ha desplazado también a las empresas productivas, poniendo en peligro su carácter de bien público, su naturaleza y el desinteresado servicio que debe ofrecerle a la humanidad mediante el conocimiento generado con la indagación universitaria.

Contar con un conocimiento que trasciende fronteras y periferias, soporta nuevos y diferentes retos a la educación universitaria contemporánea. La globalización del conocimiento debe abordarse como un proceso válido que involucra a las instituciones educativas, en especial a la totalidad de entidades educativas universitarias. No obstante, tal globalización ${ }^{12}$ en ocasiones oculta procesos de corporativización ${ }^{13}$ del conocimiento respaldada por fundamentación, desarrollo y perfeccionamiento académicos, con el riesgo de tener un mayor control de resultados surgidos de investigaciones universitarias y aprovechadas por parte de las organizaciones de transacción comercial de bienes o servicios, sobre todo organismos transnacionales. La universidad —con sus

12 Se sugiere una consideración procesual pluridimensional, motivada y fortalecida por el rápido avance de la cibernética, las tecnologías de la información y las comunicaciones. No obstante, es por el desarrollo de la globalización económica y financiera como todas las demás dimensiones de la mundialización se dan y el carácter asimétrico las determinan: una real y deseada sociedad global es afectada por una economía global debido a una creciente inequidad entre las naciones y comunidades en el interior de ellas. Es un fenómeno distante, limitador de desarrollo y extraño para la mayor parte de la población mundial.

13 En relación con las organizaciones comerciales de naturaleza privada. 
equipos, grupos y semilleros de investigación- debe seguir siendo fiel a su propia esencia, la de ser sitio de búsqueda desinteresada de saberes y conocimientos.

De igual manera, las cambiantes tecnologías de la información y la comunicación (TIC), enriquecidas con los trabajos y perfeccionamientos aportados por las llamadas teorías y ciencias de la complejidad, originan significativos cambios culturales, relacionados con la hipervalorada cultura informática. La noción de realidad coexiste con la posibilidad de construir realidades virtuales, aspecto que proyecta múltiples, diversos y también, cambiantes provocaciones para la educación superior.

Las realidades y transformaciones que transcurren en la actualidad hacen posibles una diversidad de elementos conocidos en ámbitos científicos e intelectuales como las megatendencias de la sociedad contemporánea, los cuales supuesta y abiertamente impactan los conocimientos académicos.

Así pues, de acuerdo con el pensamiento complejo (Morin, 1994b), el cual implica un cambio de pensamiento, el estudio de los fenómenos culturales-educativos puede hacerse desde la dependencia de dos perspectivas: holística o reduccionista. La primera se refiere al abordaje desde el todo o todo-múltiple; y la segunda, a un estudio simplista-reduccionista desde las partes. Para considerar las incidencias que desde otros discursos próximos o lejanos se hacen al sistema educativo, la complejidad circula ineludiblemente.

Retomando el caso de los discursos, desde el ámbito de las ciencias de la actividad física y con ella las prácticas recreativas, por ejemplo, el discurso desde las ciencias médicas que se viene concibiendo ha sido normalizado desde una perspectiva simplificadora; tornándose en ocasiones en discurso gubernamental que reduce la complejidad con miras a la educación, lo anterior, tanto desde ópticas político-ideológicas liberales como conservadoras. Así se viene asumiendo la recreación hasta el momento, por ejemplo en el caso mencionado, utilizada en la mayoría de instituciones, sobre todo escolares, como fin en dichas entidades, siendo contaminadas por tales discursos.

Las prácticas de aprendizaje y fomento que se generan en la institución escolar son instrumentos técnicos considerados irreemplazables para el establecimiento y consecución de propósitos, ya sea por la valoración de contenidos evidentes que crean, transfieren y reforman, o por los procesos y procedimientos de participación que facilitan, estimulan y mantienen.

En el marco de las relaciones, factor clave del paradigma emergente, las interacciones, de acuerdo con Maturana (1999) "resultan de las coordinaciones conductuales" (s.p.) de los miembros que integran un sistema social, las retroacciones, las cuales se dan en torno a un proceso de cambios ocurridos en los ecosistemas o que pueden crearse generando una reacción ante determinada perturbación, y por tanto, ocurre que una transformación conlleva a variaciones del estado inicial. Tales aspectos son probables de ocurrir alrededor de la pedagogía y es necesario considerar sus características, posibilidades, vínculos y diferencias entre ellas (Maturana, 1999).

Puesto que la pedagogía se ocupa de disponer mediante sus funciones la formación de la persona para la sociedad y las culturas, desde hace un buen tiempo ha sido reiterativo asumir y tener una marcada predisposición a reducir la pedagogía a la didáctica; donde los desempeños educativos son considerados como procedimientos de instrumentalización técnica y metodológica.

Contrario a la acostumbrada didactización de su ejercicio ocupacional, quien se ocupa del rol pedagógico tiene entre sus cuantiosas reflexiones, indagaciones y apuestas a partir de la pregunta qué y cómo educar; también debe cuestionarse sobre por el qué y para qué educar. Como profesional, su propósito emergente desde la responsabilidad social consciente es desarrollar cualidades morales, éticas y políticas en la ciudadanía.

Toda reflexión crítica próxima con a la pedagogía de la recreación, debe posibilitar un conjunto de aspectos, elementos o situaciones conducentes al planteamiento de emergencias epistemológicas, alterativas, alternativas e innovadoras, que inviten a la apropiación responsable de problemáticas estrictamente cognoscitivas (o técnicas) asociadas con el sistema cultural, educativo y social.

Por tanto, es útil la formulación de preguntas esenciales, pertinentes y convenientes con los desarrollos científicos, sociales, humanos y ecológicos necesarios para las sociedades interculturales: ¿Qué nueva lógica es necesario considerar, comunicar y establecer para posibilitar tales desarrollos? ¿En las prácticas formativas se establecen relaciones desde lo emocional? ¿En las prácticas educativas se expresan relaciones de poder?, 
¿y en particular en aquellas en que intervienen las prácticas recreativas? ¿Qué efectos de poder se producen sobre los sujetos y producen a los sujetos? ¿Qué valores se identifican, intervienen y relacionan que conlleven a que se piense la educación?.

La búsqueda por lograr la democratización escolar forma parte de la probabilidad (posible o imposible) de establecer desde el presente un futuro perdurable por constituirse. Las instituciones (en especial la escolar) y con ellas los contextos y las dinámicas pueden transformar las injusticias e iniquidades (diferenciación social) y generar un espacio para la transformación social y cultural sustentable -intercultural- (alternativa, imaginaria pero que construye conciencias, establece sentidos y forma los cuerpos y las emociones de las personas) (Maturana, 1999).

Ante a los valores que se le reconocen a la recreación, resulta pertinente la formulación de preguntas como: ¿Para qué sus educadores o para qué las instituciones escolares? Su reflexión, ocupación y desarrollo estimula a que se consideren tres ámbitos de proyección:

- La recreación y sus prácticas culturales y educativas como fenómeno y derecho social ${ }^{14}$, elemento, principio y derecho fundamental y su establecimiento en la educación como factor primordial en los procesos de formación integral de la persona; efectos, fenómenos asociados.

- La recreación y sus prácticas culturales y educativas como componente crítico de una institución patriarcal, pero que busca transformar mediante sus dinámicas relacionales y de convivencia (ideologías), organizacionales y sociales valorando positivamente las manifestaciones emocionales individuales o grupales.

- La recreación y sus prácticas culturales y educativas como mediación de aprendizajes significativos.

14 Reconocida como tal por la Organización de las Naciones Unidas a través de la Carta Internacional de la Educación Física y el Deporte (1978). En Colombia consagrada en la Constitución Política de Colombia (artículo 52) y desarrollada en la Ley 181 de 1995.

\section{LA RECREACIÓN Y SUS PRÁCTICAS COMO FENÓMENO SOCIAL Y CREACIÓN (CULTURAL) HUMANA}

La recreación en el último tiempo ha formado parte de las culturas motrices y en la actualidad representa una multiplicidad de significaciones para las organizaciones y comunidades y es una práctica social de amplia aceptación acorde con los procesos evolutivos y madurativos de la humanidad; podría afirmarse que se ha desarrollado con las sociedades, tiene una configuración plural de prácticas, practicantes y consumidores ${ }^{15}$.

Unida a otras creaciones y prácticas culturales desde sus orígenes ha estado alejada de principios democráticos y participativos: siendo en gran medida excluyente, diferenciadora e inequitativa. La recreación ha estado acompañada de otras variadas actividades y prácticas físico-motrices mucho más armónicas e integrales. Forma parte del multipluriverso de manifestaciones corpóreas y motrices, es decir, de las culturas: física (corpórea), motrices (corporalidades o de movimientos), técnicotecnológica y otras desarrolladas por todos los pueblos y sectores sociales a lo largo de la historia humana (Mauss, 2007). Como valor cultural, conforma el conjunto de bienes y derechos consagrados para la humanidad de los cuales toda persona debe complacerse en circunstancias apropiadas, favorables y vitales.

En su implementación cotidiana, a la recreación se le atribuyen añadidos culturales como: exaltación del (de los) talento(s) y desempeño individual; valoración de la persona, virtuosismo corporal; jerarquización; exclusión; superenaltecimiento del goce; consumismo; sobrevaloración a la disciplina; sumisión a la autoridad del gestor o del empresario.

La coordinación de la recreación escolar formativa (infantil, juvenil y social comunitaria) en los clubes y organizaciones recreo-deportivas cada vez se asemeja más a la lógica de la cultura del bienestar, en la que el producto final es el consumidor que invierte dinero o recursos, y la materia prima es la niñez y la familia quienes consumen en ese proceso de comercialización. Los medios de comunicación con su poder e influencia en la cultura del consumo irracional, resaltan y dan prensa solo a aquello que favorece la utilidad productiva

15 En su contemplación o práctica algunos la valoran como ocio, pasatiempo o uso del tiempo libre. Para otros, forman parte de su forma de vida. 
y mercantilización de bienes y servicios. Ello provoca inequidad, fragmentación y exclusión, imagen distorsionada de sí mismo y cada vez más separación entre unos privilegiados que asisten y otros que no lo hacen; se convierten en meros consumidores del fenómeno recreativo y de las culturas física, motriz, deportiva y del espectáculo en general.

Las caracterizaciones contemporáneas de la recreación y las prácticas recreo-deportivas resultan ser contradictorias y potencialmente impactantes, en torno a la pérdida de valores humanistas, comunitarios y colaborativos. Las acciones, conductas y conocimientos tanto corpóreos, como motrices conforman adelantos culturales necesarios de ser salvaguardados mediante estrategias de promoción, divulgación, masificación, organización y fomento con las que se involucren para su participación activa a las jóvenes generaciones.

\section{LA RECREACIÓN COMO TRANSFORMADORA DE ORDEN SOCIAL}

La mirada, la atención, la palabra y la legitimación de la persona misma en los contextos de interacción e interrelación con las otras posibilitan marcos relacionales en los cuales múltiples formas de convivencia se enriquecen y fortalecen favoreciendo encuentros/ desencuentros constitutivos de humanidad (relaciones éticas, estéticas, políticas, ecológicas, espirituales, educativas, culturales, etc.), mediado por consideraciones de solidaridad, cooperación y armonía en pro de un marcado respeto hacia la diversidad individual y colectiva (multidimensional), la equidad y justicia social planetaria aspectos en los cuales la recreación debería nutrirse buscando caracterizar su propia naturaleza.

El juego - como aspecto esencial en el ser humanoen sus diversas manifestaciones cumple en cualquier etapa del desarrollo humano un papel importante en la configuración social contextualizada en un marco de autonomía, libertad y creatividad que conlleva distintas formas de ser, sentir, pensar y actuar. El juego es por tanto una paradoja que se revela en los ámbitos, individual, grupal y comunitario, pero también se mimetiza en ellos.

El juego y las prácticas recreativas como manifestaciones y acciones consensuadas dadas desde estrategias dialógicas (verdadera comunicación) son constitutivas en esencia de un conjunto de relaciones, interacciones y retroacciones entre quienes intervienen en dicho juego relacional, haciendo posible la presentación de opciones educativas basadas en el respeto consigo mismo, y en el reconocimiento del sí mismo en el otro (legitimación del otro) (Maturana y Nisis, 1997). Con esto se permite la construcción de una cultura donde la convivencia (Maturana, 2005) es el resultado de una perspectiva necesaria para que las polaridades (ley de opuestos) y sus conexiones tomen autoconciencia (Krishnamurti, 1996) que todo entorno es posibilitador de cambios, pues la realidad construida para la afirmación como seres humanos solo es posible desde el intercambio.

De igual manera, desde los contextos educativo, recreativo e intercultural, es fundamental indagar sobre las fortalezas aprovechadas por las mediaciones comunicativas globales, que asumen un rol preponderante en las sociedades actuales, instruyendo amplias poblaciones de manera más eficiente para efectos de procesos de transformación social, siendo pertinente considerar su capacidad formativa e integradora organizacional.

De esta forma, se requiere examinar críticamente las instituciones existentes en donde la recreación se desarrolla, implementa, existe, se reproduce (clubes, cajas de compensación familiar y organizaciones escolares). En la actualidad, todo lo que involucra la recreación debe ser considerado como megatendencia ${ }^{16}$, la cual resulta de establecer vínculos con las mediaciones comunicativas globales, como fuerza explicativa considerada desde la lógica de realidad paradigmática emergente.

Por tanto, se requiere una educación -interculturaltransformadora donde lo importante sea el nuevo aprendizaje para la concepción de hombre y mujer como seres diferentes pero interdependientes, como seres humanos con capacidades para desarrollar una sociedad centrada en la afiliación (Eisler, 1990), no en la disociación y el egoísmo sembrados a través de los distintos sistemas instaurados por el mismo ser humano en la humanidad.

Se necesita una educación que vuelva a retomar la perspectiva matrística (Maturana, 2003) de afiliaciones, donde la propuesta educativa sea reconocer la necesidad de cada ser humano (el otro como otro involucrado y

16 Se caracteriza por cambios considerables relacionados con multiplicidad de áreas (económicas, políticas, sociales, demográficas, jurídico-legales, geográficas, institucionales y tecnológicas) las cuales influyen por periodos considerables de tiempo (10 o más años) a partir de actitudes, creencias, valores y acciones asumidas al modificar e impactar sustancialmente la vida cultural de las personas. 
que involucra) gracias a la cooperación, la participación, el juego, la alegría, el compartir, el descubrimiento, la creatividad, al cuidado del otro, a la construcción de una forma de comprensión que facilite las prácticas de la religación, la magnanimidad y el perdón (Morin, 2003).

En un sentido extenso, la educación se considera como el conjunto de operaciones que se establecen alrededor de un sistema constituido por modos de coordinaciones conductuales consensuales (Maturana, 2009) modos de un grupo social visible en las interacciones y retroacciones, entre los procesos de relación y reproducción social, el sostenimiento de lo ya estructurado, tanto de sus procesos como procedimientos de transformación resultantes de la transformación.

Por tanto, los procesos de transformación intercultural, especialmente en nuestra América, conducentes a la instauración de conductas de desprendimiento en relación con aquellas creencias e ideologías que se constituyen e instalan en ciertas comunidades humanas, deberían movilizar sus conciencias, acciones y discursos educativos dentro de una lógica emancipatoria, decolonial y de profunda ecología humana.

\section{REFERENCIAS BIBLIOGRÁFICAS}

Boff, L. y Muraro. R. (2004). Femenino y masculino. Una nueva conciencia para el encuentro de las diferencias. Madrid: Trotta.

Eisler, R. (1990). The chalice and the blade, our history, our future. Nueva York: Harper \& Row.

Harding, S. (1996). Ciencia y feminismo. Madrid: Morata.

Kosko, B. (2000). El futuro borroso o el cielo en un chip. Barcelona: Editorial Crítica.

Krishnamurti, D.B. (1996). Más allá del tiempo. Barcelona: Kairos.

Maturana, H. (2009). La realidad ¿objetiva o construida? (ii): Fundamentos biológicos de la realidad. Madrid: Anthropos.

Maturana, H. y Nisis de Rezepka, S. (1997). Transformación en la convivencia. Santiago de Chile: Dolmen Ediciones.

Maturana, H. y Varela, F. (1984). El árbol del conocimiento. Santiago de Chile: Universitaria.
Maturana, H. (2005). Emociones y lenguaje en la educación y política. Santiago de Chile: JC Saez.

Mauss, M. (2007). Ensayo sobre el don. Madrid: Katz.

Max-Neef, M. (1993). Desarrollo a escala humana. Una opción para el futuro. Valparaiso: Cepaur, Fundación Dag Hammarskjöld.

Morin, E. (1994a). El método III. El conocimiento del conocimiento. Madrid: Cátedra.

Morin, E. (1994b). Epistemología de la complejidad. En: F. Schitman (ed.). Nuevos paradigmas, cultura y subjetividad (pp. 421-442). Buenos Aires: Paidós.

Morin, E. (2003). El método V. La humanidad de la humanidad. La identidad humana. Trad. Ana Sánchez. Madrid: Cátedra Teorema.

Wilber, K. (1987). Paradigma holográfico. Barcelona: Kairós. 